Izquierdo Rus, T., Escarbajal Frutos, A. y Latorre Román, P. A. (2016). Motivaciones que condicionan la formación y previenen la exclusión social de los futuros educadores. Revista de Investigación Educativa, 34(2), 385-397.

DOI: http://dx.doi.org/10.6018/rie.34.2.238381

\title{
Motivaciones que condicionan la formación y previenen la exclusión social de los futuros educadores
}

\section{Motivations that condition the training and prevent social exclusion of future educators}

\author{
Tomás Izquierdo Rus*,Andrés Escarbajal Frutos** y Pedro A. Latorre Román*** \\ *Universidad de Murcia \\ ***niversidad de Murcia \\ ****Universidad de Jaén
}

\begin{abstract}
Resumen
Este artículo tiene como objetivo explorar las motivaciones de los estudiantes universitarios en la elección de las diversas carreras de Educación. Desde un enfoque metodológico cuantitativo se presentan los datos de 391 estudiantes universitarios de la Universidad de Murcia, utilizando como instrumento de recogida de datos el cuestionario de motivos en la elección de la carrera junto al componente exclusión social. Los resultados mostraron diferencias en la titulación elegida y situación laboral del estudiante. Se concluye que los estudiantes eligen cursar una carrera universitaria relacionada con la educación para evitar la exclusión socioeconómica y laboral o por vocación. El artículo finaliza con propuestas de intervención socioeducativas y nuevas líneas de investigación en el área.
\end{abstract}

Palabras clave: educadores; exclusión social; motivaciones; universidad.

\begin{abstract}
This paper aims to explore the motivations of university students in career choice. Adopting a quantitative methodological approach, this study presents data of 391 university's students of the University of Murcia using as the data collection instrument the questionnaire of motives in career choice, together with the social exclusion scale. Results showed differences in the

Correspondencia: Tomás Izquierdo Rus, Departamento de Métodos de Investigación y Diagnóstico en Educación, Facultad de Educación, Universidad de Murcia, 30100 Murcia (España). E-mail:tomaizq@um.es
\end{abstract}


student's career choice and the situation on the labor market. It concludes that the students choose a career on education to avoid the risk of socioeconomic and labor exclusion or vocation. The paper concludes with proposals for social educational intervention and new lines of research in this area.

Keywords: educators; motivations; social exclusion; university.

\section{Introducción}

No se niega que la mundialización y la globalización han supuesto oportunidades profesionales y de negocios inimaginables para muchas personas, pero también han provocado cambios económicos y sociales tan vertiginosos y tan difíciles de digerir por la gran mayoría de las poblaciones que han acrecentado la pobreza, la vulnerabilidad y la exclusión social en espacios, grupos y personas impensables hace unas décadas. En el contexto europeo, en la Europa de las dos velocidades, millones de personas se han visto abocadas al desempleo, formando una cohorte de precarizados que han tenido que abandonar sus tradicionales sistemas de vida, su inclusión en los círculos sociales (Bauman, 2003/2005; Beck, 2000/2002; Escarbajal, 2010; Stiglitz, 2002/2003).

De este modo, se puede encontrar en las ciudades, cada vez más, a grupos excluidos no directamente vinculados a la variable insuficiencia de renta. Por eso, el tradicional concepto de pobreza no puede abarcar las nuevas situaciones que están emergiendo en los núcleos urbanos (Gomà, 2012). Hoy se tendría que hablar de exclusión social como de un proceso por el que ciertos grupos y/o personas tienen dificultades de acceso a los bienes y servicios de una sociedad, al tiempo que esta situación les impide vivir de manera autónoma en el contexto en el que habitan. De esta concepción es evidente que, entre otras conclusiones, se debe inferir que la exclusión social es poliédrica, y las situaciones que la determinan pueden variar en función de circunstancias educativas, sociales y políticas y, por supuesto, económicas (Castro y Cano, 2013). Por esta razón en un mundo donde la globalización, el neoliberalismo y el pensamiento único han creado bolsas de marginación y exclusión social, fundamentalmente entre los jóvenes, se debe tener confianza en la educación como palanca impulsora de la movilidad sociolaboral ascendente y de los procesos democratizadores de inclusión social.

Así, la exclusión ha pasado a ser un sustituto de la antigua explotación porque los vínculos entre explotadores y explotados son completamente diferentes de los que se establecen entre incluidos y excluidos. En otro orden de cosas, el 30\% de los estudiantes universitarios cambia o abandona la carrera. Este fracaso escolar, además de significar un considerable gasto para las arcas públicas, supone elevados niveles de frustración para esos jóvenes, que o bien no fueron adecuadamente orientados a la hora de elegir carrera o no encontraron en las elegidas las expectativas que tenían al comenzar sus estudios (Peña, 2010). Y, como se ha señalado anteriormente, tanto la pobreza como la exclusión no sólo deben ser medidas hoy en términos económicos, sino teniendo en cuenta otras variables como la formación o el acceso a los servicios básicos de una comunidad (Sánchez, 2013); y, en ese sentido, algunos estudios (Beck, 2012/2012; Colectivo IOÉ, 2013; Estefanía, 2011; Oxfam Intermón, 2014; Stiglitz, 2012/2012) alertan de que la situación en España puede agravarse al generar más desigualdad y pobreza. 
Y esa situación no es consecuencia de la falta de crecimiento económico ni de formación, pues muchos de los jóvenes españoles que se han visto obligados a emigrar tienen título universitario; es consecuencia de la lógica del sistema capitalista, un sistema injusto que convierte a un porcentaje considerable de la población en excluidos; son jóvenes, en su mayoría, que no han tenido aún acceso a un trabajo o lo han perdido, jóvenes que regresaron al hogar familiar porque no podían seguir pagando facturas y letras acumuladas, toda una generación que corre el riesgo de quedarse sin futuro (Bauman, 2008/2010). Por eso ha escrito García Roca (2012) que la actual crisis actúa como un tobogán que empuja hacia abajo y hacia fuera. Es lo que Wacquant (2004/2010) llamó marginalidad urbana avanzada, resultado del crecimiento y el progreso económico. Aumentando la vulnerabilidad y la exclusión de la clase trabajadora y reduciendo considerablemente el número de familias de la clase media que se creía estabilizada.

Otros autores (Herrero, Peláez y González, 2007) destacan cómo, en su sentido amplio, la vulnerabilidad refleja dos condiciones: la de los vulnerados (personas que ya sufren carencias efectivas, están situadas en la pobreza y tienen grandes dificultades para salir de ella) y la de los vulnerables (cuyo deterioro de vida aún no está materializado pero sí anunciado). De ahí que entiendan la vulnerabilidad social como una condición de riesgo inmediato, pero mucho más de riesgo futuro, de grupos o colectivos afectados en la satisfacción de su bienestar por las características de sus contextos sociohistóricos y culturales.

Las desigualdades sociales sabemos que son la traducción de determinados sistemas de producción. Efectivamente, en las sociedades jerarquizadas, como la nuestra, las desigualdades son consecuencia de diferentes variables que dependen del sistema social imperante y de cómo éste se reproduce. Todos los parámetros que definen la posición social de las personas (sexo, lugar de residencia, trabajo, estudios, etc.) son susceptibles de intervenir en las situaciones de desigualdad. Sin embargo, la exclusión social no es definible sólo mediante categorías que aíslan a grupos determinados de personas en función de aquellos parámetros, sino que, como se ha comprobado en la situación de crisis económica, pueden devenir por causas inesperadas o estados de incertidumbre social. Es decir, la desigualdad social ya no es únicamente la manifestación de la desigualdad entre quienes están arriba y abajo en la escala social, sino también de la cada vez más distancia existente entre los pertenecientes a la dinámica social y los que se ven expulsados de la misma. Por tanto, "la desigualdad social pasa a ser una cuestión estructural y multidimensional que tiene como una de sus consecuencias más destacadas la pérdida de la ciudadanía" (Morón y Cobacho, 2009, p. 79).

Hay algunos datos que corroboran lo anterior: el porcentaje de población en riesgo de pobreza aumentó al 22,2\% y el 16,1\% de los hogares españoles manifestó llegar a fin de mes con "mucha dificultad" (Instituto Nacional de Estadística [INE], 2014); destacar que la tasa de riesgo de pobreza difiere en función del nivel de formación del individuo; y, en la actualidad, la tasa de riesgo de pobreza en 2014 se situó en el 22,2\% de la población residente en España, frente al 20,4\% registrado el año anterior. En el caso de la Región de Murcia el ingreso medio anual neto por persona fue de 7.536 euros y la tasa de riesgo de pobreza se situaba de un $26.4 \%$, muy alejado del $1.9 \%$ de la Comunidad Madrid o del 16.6\% de Cataluña. Las regiones de Andalucía (24.3\%), Murcia (22,7\%) y Galicia (20,8\%) fueron en 2014 las comunidades autónomas 
con mayores porcentajes de hogares que llegaban a fin de mes con "mucha dificultad". En el caso de Murcia, durante el 2014, el 55.6\% de los murcianos no tuvieron capacidad para afrontar gastos imprevistos y el $61.5 \%$ de hogares murcianos no podían permitirse ir de vacaciones fuera de casa al menos una semana al año (INE, 2014).

A este respecto, un estudio cualitativo (con una muestra de 1.000 jóvenes de españoles entre 18 y 24 años) concluyó que un 48.6\% de los entrevistados aceptaría cualquier trabajo sin importar el lugar ni el sueldo, el 79.2\% ve muy elevada la probabilidad de tener que estudiar más, y el $84.9 \%$ tener que trabajar "en lo que sea"; y, en el mismo estudio, un $56.6 \%$ cree que las personas con mayores niveles de estudios tienen mejores oportunidades de conseguir buenos trabajos (Rodríguez y Ballesteros, 2013). Por eso se quiere comprobar en el estudio que se presenta si los jóvenes que eligen una titulación en educación lo hacen por vocación o para conseguir una estabilidad socioeconómica y laboral que les evite ser excluidos en el futuro.

Es un hecho la inestabilidad económica en Europa y que tuvo un impacto negativo en el descenso de la emancipación residencial de los jóvenes y en el ritmo de creación de nuevos hogares, pues la tasa de paro tiene unos valores muy altos entre los jóvenes, sobre todo entre los españoles, llegando al 56.5\% entre los españoles menores de 25 años, una tasa de desempleo juvenil sólo superada por Grecia en la Unión Europea. Además, los llamados ninis (jóvenes que ni estudian ni trabajan), son un $5.6 \%$ más en España que la media Europea. Ello provoca exclusión, aumento de la desigualdad e inestabilidad (Consejo Económico y Social [CES], 2013) y enfatiza que la crisis económica ha afectado más a unos colectivos (en este caso los jóvenes con poca formación) que a otros. En la Región de Murcia, los jóvenes menores de 30 años experimentan unas tasas de desempleo del $37.3 \%$. Son jóvenes vulnerables y con altas tasas de riesgo de exclusión social, sobre todo los que abandonaron el sistema educativo sin haber obtenido una titulación básica (Consejo Económico y Social de la Región de Murcia [CESRM], 2012).

La prolongación de la situación anterior en el tiempo, en el contexto español, está provocando un aumento de la desigualdad en nuestro país (Organización para la Cooperación y Desarrollo Económico [OCDE], 2011), deteriorando el capital humano; y los que tienen una buena cualificación profesional y no encuentran un empleo están emigrando; y se sabe que esos jóvenes son el activo más valioso de la sociedad (Stiglitz, 2012/2012). Pero, siendo el activo más preciado, no parecen tenerlo fácil para superar la situación de exclusión y marginación, pues así lo demuestra una investigación longitudinal de cinco años (Quintini y Manfredi, 2009) que analizó la transición de la formación al mundo laboral de jóvenes de EEUU y de 12 países de la UE; destacando, de entre sus resultados, que los jóvenes españoles (junto con algunos jóvenes de los países de Europa del sur) son quienes tardan más tiempo en encontrar un empleo desde que terminan la formación. Este estudio corrobora la difícil situación que actualmente viven los jóvenes residentes en España.

Para combatir el desempleo y la exclusión socioeconómica y laboral, los jóvenes que no pueden emigrar parece que tienen dos opciones: la primera es seguir aceptando empleos por debajo de su nivel de cualificación, por aquello que auguraba Marx de que, si no aceptan esos empleos, existe un ejercito en la reserva dispuesto a trabajar y aceptar las condiciones que ellos puedan rechazar; la segunda opción es apostar por la 
formación, la máxima posible, pero, en esta segunda opción, la duda también les asalta: ¿Debo estudiar por vocación o pensando en salidas profesionales? Evidentemente, la vocación es fundamental a la hora de elegir carrera, pero en las actuales circunstancias socioeconómicas los jóvenes parecen mirar más a las posibles oportunidades de empleo de una carrera. Esto es corroborado por el estudio de Alonso, Chavarri, Poy, Sancho y Chamorro (1997) que concluye que el $25 \%$ de los alumnos no eligen carrera vocacional por las dificultades de acceso al mundo laboral.

En la línea anterior, otro estudio (Avendaño y González, 2012) analizó las motivaciones de los alumnos universitarios de primer curso en diferentes especialidades de Educación. En esa investigación se concluye que los jóvenes no sólo eligen una carrera en Educación por motivos vocacionales, sino por las perspectivas futuras de alcanzar empleo. En otro trabajo, elaborado por el Circulo de Formación a partir de los datos extraídos del Salón de orientación universitaria de UNITOUR 2013-2014, se afirmaba que el $40 \%$ de los jóvenes españoles elegían una carrera por vocación, mientras que el 36\% lo hacía en virtud de las posibles salidas profesionales (Géniz, 2014).

La evidencia de los estudios aportados demuestra que los estudiantes están convencidos de que las características del sistema educativo influyen de manera decisiva en la movilidad social ascendente. Tener una carrera, en este caso en Educación, promociona expectativas positivas de un futuro trabajo estable que aleje de la exclusión y marginación a la que se ven sometidos cientos de miles de jóvenes españoles. Por ello, el objetivo general de la presente investigación ha sido analizar si la exclusión social ha sido un elemento motivador de los estudiantes universitarios de la Región de Murcia en la elección de los Grados en Educación y, con un carácter más específico, mostrar diferencias en función de variables como el sexo, la titulación y la situación laboral.

\section{Metodología}

\section{Participantes}

En esta investigación participaron 391 estudiantes de la Facultad de Educación de la Universidad de Murcia, matriculados en los diferentes grados y licenciaturas impartidas durante el curso académico 2012/2013 y 2013/2014. Según el sexo, el 78.8\% son mujeres y el $21.2 \%$ son hombres y la media en edad es de 21.40 años $(\sigma=4.66)$. Por titulaciones los participantes se agruparon de la siguiente forma: 16.1\% fueron estudiantes de Educación Social, 27\% de Pedagogía, 27\% de Educación Primaria, $18.1 \%$ de Educación Infantil y el $11.7 \%$ de Psicopedagogía. Tan sólo el 12\% de los participantes se encontraba trabajando en el momento de la investigación, frente al $88 \%$ que no tenían trabajo.

\section{Instrumento}

Se utilizó el cuestionario sobre motivos en la elección de la carrera universitaria de Gámez y Marrero (2003), adaptando las preguntas que aludían a otras titulaciones por las de Educación. El cuestionario se compone de 45 ítems en una escala tipo Likert 
con opciones desde 1 (nada) hasta 5 (mucho). Cada ítem expresa una afirmación de la forma Porque quieres tener un nivel social y económico adecuado donde se van presentando diferentes temas relacionados con las metas, motivos de logro, poder, afiliación, problemas personales, búsqueda de conocimientos y motivación extrínseca. La fiabilidad de la prueba es muy elevada $(\alpha=.891)$, que indica que el instrumento posee una adecuada consistencia interna. Como medida de la exclusión social se utilizaron 4 ítems que hacen referencia a la estabilidad, posición social y nivel económico. El cuestionario se complementa con una serie de preguntas generales relacionadas con la edad, sexo, titulación, curso, situación laboral, etc.

\section{Procedimiento}

Los estudiantes participaron de forma voluntaria en la investigación, obteniendo los datos en las aulas donde habitualmente recibían sus clases. En la recogida de información colaboraron becarios de la Universidad de Murcia, que participaron de forma activa en la realización de la investigación y con formación específica para la recogida de información. Previo a la cumplimentación del cuestionario, a los estudiantes participantes se les informó del propósito del estudio y se les garantizó el anonimato así como el uso confidencial de la información obtenida. La realización del cuestionario tuvo un tiempo promedio de 30 minutos por estudiante.

\section{Análisis de datos e interpretación de resultados}

Los datos de este estudio se han analizado mediante el programa estadístico SPSS v.22 (IBM SPSS Statistics for Windows, NY) y el nivel de significación se estableció en p<.05. Los datos extraídos del cuestionario se analizaron utilizando la misma solución factorial propuesta por los autores del instrumento, que consiste en cinco factores: superación de problemas afectivos, logro y prestigio, logro y afiliación, poder y motivación extrínseca. El análisis de fiabilidad por factores ofreció un alpha de Cronbach de .798 para el componente poder, de .776 para el componente superación, de .780 para el componente afiliación, de .696 para el componente logro y .640 para el componente motivación. Con respecto a la escala exclusión social, el alpha de Cronbach resultante fue de .747.

Como se puede observar en la tabla 1, la puntuación de las mujeres es ligeramente mayor que la de los hombres en cada uno de los factores, si bien al aplicar la prueba $t$ $(\mathrm{p}<.05)$ estas diferencias no llegan a ser significativas. Probablemente estos resultados estén marcados por la predominancia del sexo femenino en las titulaciones de Educación.

En la tabla 2 se muestra cada uno de los factores en relación a la titulación. Los estudiantes de Pedagogía destacan en poder, superación, afiliación, motivación y exclusión. Eligieron la carrera atendiendo a motivos de afiliación por la necesidad de sentirse aceptados en el grupo y la preocupación por el establecimiento de nuevas relaciones. Otros motivos están relacionados con el poder, que significa ejercer la titulación de Pedagogía como un mecanismo que permite influir sobre las decisiones de los demás, metas claras de superación personal, motivaciones de carácter externo (influencia de familiares, amigos o iguales) y, por último, disponer de una profesión que les permita obtener un reconocimiento social y les aporte beneficios de estabilidad y de carácter 
económico. Sin embargo, los estudiantes de Educación Primaria destacan en logro, buscando posicionarse en la sociedad, emprender un camino con esfuerzo y dedicación y evitar la sensación de fracaso.

Por el contrario, los estudiantes de Educación Infantil presentan menor puntuación en poder, los estudiantes de Educación Primaria en superación, los estudiantes de Psicopedagogía en afiliación y logro, por último los estudiantes de Educación Social en motivación y exclusión.

Tabla 1

Diferencias según sexo en la elección de la carrera

\begin{tabular}{llcccccc}
\hline Sexo & & Poder & Superación & Afiliación & Logro & Motivación & Exclusión \\
\hline \multirow{2}{*}{ Mujer } & Media & 32.26 & 17.42 & 41.39 & 29.60 & 22.29 & 12.91 \\
& Desv. típ. & 7.291 & 5.903 & 6.390 & 5.004 & 5.389 & 3.84 \\
\multirow{2}{*}{ Hombre } & Media & 32.05 & 17.06 & 40.19 & 28.73 & 22.39 & 12.68 \\
& Desv. típ. & 7.517 & 5.653 & 6.275 & 4.591 & 4.571 & 4.00 \\
\multirow{2}{*}{ Total } & Media & 32.22 & 17.35 & 41.13 & 29.42 & 22.31 & 12.85 \\
\multirow{2}{*}{ Sig. } & Desv. típ. & 7.331 & 5.845 & 6.376 & 4.926 & 5.220 & 3.87 \\
\hline
\end{tabular}

Tabla 2

Diferencias según titulación en la elección de la carrera

\begin{tabular}{llcccccc}
\hline Titulación & & Poder & Superación & Afiliación & Logro & Motivación & Exclusión \\
\hline \multirow{2}{*}{ Ed. Social } & Media & 30.35 & 17.44 & 41.11 & 28.79 & 20.79 & 11.55 \\
& Desv. est. & 7.475 & 5.719 & 6.444 & 4.952 & 5.197 & 3.946 \\
\multirow{2}{*}{ Pedagogía } & Media & 35.31 & 20.48 & 42.80 & 29.89 & 24.45 & 14.01 \\
& Desv. est. & 7.654 & 6.459 & 6.480 & 5.691 & 5.062 & 3.521 \\
\multirow{2}{*}{ Ed. Primaria } & Media & 32.04 & 15.49 & 41.14 & 30.16 & 22.03 & 12.95 \\
& Desv. est. & 6.865 & 4.143 & 5.804 & 4.440 & 4.884 & 4.000 \\
Ed. Infantil & Media & 29.99 & 16.15 & 39.92 & 29.21 & 21.67 & 11.94 \\
\multirow{2}{*}{ Psico- } & Desv. est. & 7.355 & 5.978 & 6.210 & 4.256 & 5.669 & 4.028 \\
pedagogía & Media & 31.41 & 16.13 & 38.85 & 27.48 & 21.15 & 13.19 \\
\multirow{2}{*}{ Total } & Desv. est. & 4.787 & 4.861 & 7.140 & 5.014 & 4.190 & 3.208 \\
& Media & 32.21 & 17.34 & 41.09 & 29.38 & 22.32 & 12.85 \\
& Desv. est. & 7.326 & 5.839 & 6.427 & 4.974 & 5.215 & 3.872 \\
\hline
\end{tabular}

Al comparar las medias de la titulación respecto a la elección de la carrera, mediante la prueba ANOVA, se muestran diferencias estadísticamente significativas $(\mathrm{p}<.05)$ en todos los factores, tal y como se observa en la tabla 3. 
Tabla 3

Diferencias según titulación en la elección de la carrera

\begin{tabular}{llccccc}
\hline \multicolumn{2}{c}{ FACTORES } & Suma de cuadrados & Gl & Media cuadrática & F & Sig. \\
\hline Poder & Inter-grupos & 1608.657 & 4 & 402.164 & 8.035 & .000 \\
& Intra-grupos & 19268.933 & 385 & 50.049 & & \\
& Total & 20877.590 & 389 & & & \\
Superación & Inter-grupos & 1563.010 & 4 & 390.752 & 12.856 & .000 \\
problemas & Intra-grupos & 11732.750 & 386 & 30.396 & & \\
afectivos & Total & 13295.760 & 390 & & & \\
Afiliación y & Inter-grupos & 636.540 & 4 & 159.135 & 3.970 & .004 \\
logro & Intra-grupos & 15471.327 & 386 & 40.081 & & \\
& Total & 16107.867 & 390 & & & \\
Logro & Inter-grupos & 281.823 & 4 & 70.456 & 2.904 & .022 \\
(eficacia) & Intra-grupos & 9390.542 & 387 & 24.265 & & \\
& Total & 9672.365 & 391 & & & \\
Motivación & Inter-grupos & 730.164 & 4 & 182.541 & 7.134 & .000 \\
extrínseca & Intra-grupos & 9876.874 & 386 & 25.588 & & \\
& Total & 10607.038 & 390 & & & \\
Exclusión & Inter-grupos & 313.968 & 4 & 78.492 & 5.474 & .000 \\
social & & & & & & \\
& Intra-grupos & 5535.295 & 386 & 14.340 & & \\
\hline
\end{tabular}

La elección de la carrera también está determinada por la situación laboral del estudiante. Aplicando la prueba t se observa tan sólo diferencias estadísticamente significativas en la escala exclusión social (Tabla 4). Esto puede deberse a que las personas participantes que se encuentran trabajando, en muchas ocasiones, no tienen un empleo estable y realizan trabajos a tiempo parcial y con baja remuneración económica.

Tabla 4

Diferencias según situación laboral en la elección de la carrera

\begin{tabular}{|c|c|c|c|c|c|}
\hline \multicolumn{2}{|c|}{ ¿Se encuentra trabajando actualmente? } & \multirow{2}{*}{$\begin{array}{c}\text { Media } \\
32.72\end{array}$} & \multirow{2}{*}{$\begin{array}{c}\text { Desv. } \\
5.7477\end{array}$} & \multirow{2}{*}{$\begin{array}{c}\text { Error } \\
.838\end{array}$} & \multirow[t]{2}{*}{ Sig. } \\
\hline \multirow{4}{*}{ Superación problemas afectivos } & SI & & & & \\
\hline & $\mathrm{NO}$ & 32.13 & 7.521 & .406 & \multirow[t]{2}{*}{.606} \\
\hline & SI & 17.13 & 5.007 & .730 & \\
\hline & $\mathrm{NO}$ & 17.37 & 5.949 & .321 & \multirow[t]{2}{*}{.791} \\
\hline \multirow{2}{*}{ Afiliación y logro } & SI & 41.68 & 6.217 & .907 & \\
\hline & $\mathrm{NO}$ & 41.01 & 6.459 & .348 & \multirow[t]{2}{*}{.502} \\
\hline \multirow{2}{*}{ Logro (eficacia) } & SI & 30.55 & 4.282 & .625 & \\
\hline & NO & 29.22 & 5.045 & .272 & \multirow[t]{2}{*}{.085} \\
\hline \multirow{2}{*}{ Motivación extrínseca } & SI & 21.74 & 4.489 & .655 & \\
\hline & $\mathrm{NO}$ & 22.40 & 5.308 & .286 & \multirow[t]{2}{*}{.421} \\
\hline \multirow[t]{2}{*}{ Exclusión social } & SI & 14.00 & 3.230 & .471 & \\
\hline & NO & 12.70 & 3.930 & .211 & .031 \\
\hline
\end{tabular}




\section{Discusión y conclusiones}

Cada vez son más evidentes y graves las dificultades de los jóvenes para encontrar empleo digno, con lo que se han convertido en un colectivo tremendamente vulnerable que puede verse abocado a la exclusión social. Son una generación que pasan por dificultades que ni ellos ni sus padres llegaron a imaginar. Son parte de las vidas desperdiciadas a las que aludió Bauman (2003/2005). Y, como remarcan Jiménez, Musitu y Herrero (2011), la pérdida o ausencia de empleo significa anular proyectos vitales, renunciar al control de la propia vida, a la autonomía e independencia personales y a la sensación de ser socialmente útiles. Por ello, la dificultad de acceso a un empleo digno se percibe como la puerta de entrada en la vulnerabilidad y la exclusión social.

En ese sentido, y como se indicó anteriormente, esta investigación tiene como objetivo identificar si la exclusión social ha sido un factor motivador del universitario que ingresa en las titulaciones de Educación, sobre todo en tiempos de reinvención del significado de la propia exclusión social motivada por la crisis económica, como han estudiado García Roca (2012) y Lépore (2006). Los motivos por los que un estudiante elige una titulación universitaria no siempre están relacionados con la formación que va a recibir en la Universidad sino más bien con aspectos que inciden en su desempeño posterior, así como por la posibilidad de mejorar sus condiciones de vida al desempeñarla, como pusieron de manifiesto algunas investigaciones sobre la temática (Alonso et al., 1997; Avendaño y González, 2012; Fundación Universidad Carlos III, 2012).

La necesidad de conocer estos motivos justifica las aportaciones de este estudio; por un lado, el apoyo a la fiabilidad y validez de una escala para la evaluación de las motivaciones principales de los estudiantes en la elección de los estudios universitarios siendo útil tanto a nivel investigador como a nivel práctico, pero también para que alumnado pueda conocer sus propias motivaciones y puedan reflexionar sobre ello; por otro, la identificación de una serie de motivos evaluados por este instrumento, complementado con la escala de exclusión social como otra de las motivaciones en la elección de la carrera.

Los estudiantes de la Facultad de Educación de la Universidad de Murcia que se matricularon en el curso académico 2012/2013, realizaron la elección de la carrera con diferentes motivaciones en función de la titulación elegida. En relación al sexo, los resultados de este trabajo difieren de los resultados de Gámez y Marrero (2003) que encontraron diferencias en las motivaciones de hombres y mujeres de las titulaciones de psicología, derecho y biología. Por titulación, coincidiendo con el trabajo de Gámez y Marrero (2005), se manifiestan diferencias en todos los factores analizados, siendo más marcadas en poder, superación, motivación extrínseca y exclusión social. Es decir, existen motivaciones de poder, superación de problemas afectivos al priorizar la creación y fortalecimiento de lazos afectivos, motivaciones extrínsecas como el consejo de padres y amigos y evitar la exclusión social al intentar conseguir una estabilidad socioeconómica y laboral.

Se observan también diferencias en afiliación y logro, al conceder importancia a la pertenencia a un grupo mediante la manifestación de la necesidad de ser aceptado socialmente y el sentimiento de seguridad en las relaciones interpersonales. En último lugar, la elección de la carrera también está condicionada por el deseo del estudiante 
de un empleo estable, que les garantice una posición social y económica y que les aleje de situaciones de exclusión y marginación (Arrillaga et al., 2012; Escarbajal e Izquierdo, 2013; Escarbajal, Izquierdo y López, 2014, Pieck, 2001) como grupo social vulnerable (Tezanos, 2005).

Este es el primer estudio realizado sobre las motivaciones en la elección de la carrera en la Universidad de Murcia y más concretamente en las titulaciones de la Facultad de Educación. No obstante, sería conveniente realizar nuevas investigaciones en otras titulaciones y Facultades de la Universidad para conocer si las motivaciones difieren en función de la carrera, así como estudios longitudinales que permitan indagar si las motivaciones varían durante el transcurso de la titulación elegida.

Como propuestas de actuación, los resultados obtenidos podrían ser un indicador importante del tipo de motivaciones que más valoran los estudiantes universitarios y su posible relación con otras variables como el rendimiento académico o el abandono de la carrera, con la finalidad de detectar los inconvenientes al inicio de los estudios universitarios y brindar un apoyo adecuado desde los servicios de asesoramiento y orientación de la Universidad. Además la realización de este tipo de estudios permite ofrecer no sólo un diagnóstico de los motivos que orientan al estudiante en la elección de los estudios sino también, según González (2006), en cómo se integran estos motivos en la regulación de su actuación profesional y adecuarse a las nuevas demandas que plantea el mercado laboral (Izquierdo, 2010).

La consideración de los motivos en la elección de la carrera es un tema en el que, en la actualidad, se ha avanzado mucho a nivel teórico. Sin embargo no ha repercutido en igual importancia en la investigación, dado el interés y la relevancia que tiene en la formación de los futuros educadores. Dicha relevancia se justifica al considerar que su desempeño posterior puede estar influido por otros motivos que no tienen que ver con aspectos relacionados con la formación académica. Por eso, y de acuerdo con Lépore (2006), la exclusión social deberá valorarse a la luz de las dificultades de los individuos y grupos para participar activamente en su comunidad ejerciendo la profesión para la que fueron formados; de este modo, un individuo estará excluido si habita un espacio pero no puede participar, y quisiera hacerlo, en las actividades sociales del mismo por motivos que están fuera de su control.

\section{Referencias}

Alonso, A., Chavarri, I., Poy, I., Sancho, A. y Chamorro, D. (febrero-marzo, 1997). Universidad: La guerra por el título. Trabajo presentado en el VI Congreso de Estudiantes de la Comunidad Valenciana, Sociedad multicultural: competencia y cooperación. Resumen recuperado de http://www.oc.im.ehu.es

Arrillaga, I., Pisonero, S., Arrillaga, D., Camino, I., Askunce, C., Sáenz, A. y Pérez, Z. (2012). Formación y exclusión social (Documento de Síntesis No. C20110176). Recuperado del sitio de Internet Servicio de Empleo Público Español, SEPE: http://www.sepe.es/ LegislativaWeb/verFichero.do?fichero=09017edb8011f10c

Avendaño, C. y González, R. (2012). Motivos para ingresar a las carreras de Pedagogía de los estudiantes de primer año de la Universidad de Concepción. Estudios Pedagógicos, 32(2), 21-33. doi: 10.4067/S0718-07052012000200002 
Bauman, Z. (Ed.). (2005). Vidas desperdiciadas: la modernidad y sus parias (Trad. P. Hermida). Barcelona: Paidós. (Reimpreso de Wasted lives: Modernity and its outcasts, por Z. Bauman, Ed., 2003, Nueva York, EE.UU.: Wiley)

Bauman, Z. (Ed.). (2010). Mundo consumo: ética del individuo en la aldea global (Trad. A. Santos; Vol. 175). Barcelona: Paidós. (Reimpreso de Does ethics have a chance in a world of consumers?, por Z. Bauman, Ed., 2008, Nueva York, EE.UU.: Harvard University Press)

Beck, U. (Ed.). (2002). Libertad o capitalismo. Conversaciones con Johannes Willms (Trad. B. Moreno; Vol. 100). Barcelona: Paidós Ibérica. (Reimpreso de Freedom or capitalism, por U. Beck, Ed., 2000, Frankfurt, Alemania: Suhrkamp).

Beck, U. (Ed.). (2012). Una Europa alemana (Trad. A. Valero). Barcelona: Paidós Ibérica. (Reimpreso de Das deutsche Europa, por U. Beck, Ed., 2012, Berlín, Alemania: Suhrkamp).

Castro, L. I. y Cano, R. (2013). Pobreza y vulnerabilidad: Factores de riesgo en el proceso educativo. Contextos Educativos, 16, 55-72. doi: 10.18172/con.1290

Consejo Económico y Social. (2013). Memoria sobre la situación económica y laboral en España 2012 (Informe No. 20). Madrid: CES.

Consejo Económico y Social de la Región de Murcia. (2012). El mercado laboral de los jóvenes en la Región de Murcia. Murcia: Servicio Regional de Empleo y Formación.

Colectivo IOÉ. (2013). Barómetro social de España. Madrid: IOÉ.

Escarbajal, A. (2010). Estudios de las respuestas socioeducativas ofrecidas a los inmigrantes en la región de Murcia. Revista de Investigación Educativa, 28(1), 157-170. Recuperado de http://revistas.um.es/rie/article/view/97881

Escarbajal, E. e Izquierdo, T. (2013). Percepciones psicosociales de la exclusión que determinan la inclusión sociolaboral. Revista de Ciencias Sociales, 19(1), 13-21. Recuperado de http://www.redalyc.org/articulo.oa?id=28026467007

Escarbajal, A., Izquierdo, T. y López, O. (2014). Análisis del bienestar psicológico en grupos en riesgo de exclusión social. Anales de Psicología, 30(2), 541-548. Recuperado de http://www.redalyc.org/articulo.oa?id=16731188017

Estefanía, J. (Ed.). (2011). La economía del miedo. Barcelona: Círculo de lectores.

Fundación Universidad Carlos III (2012). XVI Estudio de inserción profesional de los titulados de la Universidad Carlos III de Madrid. Promoción 2010. Recuperado de http://e-archivo. uc3m.es/bitstream/handle/10016/14286/resumen_fundacion_EIPXVI.pdf?sequence=1 .

Gámez, E. y Marrero, H. (2003). Metas y motivos en la elección de la carrera universitaria: un estudio comparativo entre Psicología, Derecho y Biología. Anales de Psicología, 19(1), 121-131. Recuperado de http://www.redalyc.org/articulo.oa?id=16701912

Gámez, E. y Marrero, H. (2005). Bases cognitivas y motivacionales de la capacidad humana para las relaciones interpersonales. Anuario de Psicología, 36(3), 239-260. Recuperado de http://www.redalyc.org/articulo.oa?id=97017406001

García Roca, J. (2012). Reinvención de la exclusión social en tiempos de crisis (Vol. 35). Madrid: Cáritas.

Géniz, D.J. (23 de abril de 2014). La vocación por encima de todo. Diario de Sevilla. Recuperado de http://www.diariodesevilla.es/article/sevilla/1757455/la/vocacion/ por/encima/todo.html 
Gomà, R. (2012). Políticas sociales, territorio y democracia. Barcelona inclusiva. RES, 14, 1-15. Recuperado de http://www.eduso.net/res/pdf/14/barnainclusiva_res_14.pdf González, V. (2006). La formación de competencias profesionales en la Universidad: Reflexiones y experiencias desde una perspectiva educativa. XXI. Revista de Educación, 8, 175-187. Recuperado de http://www.uhu.es/publicaciones/ojs/index.php/ xxi/article/view/685/1079

Herrero, V., Peláez, E. y González, L. (2007). Vulnerabilidad social, rezago y deserción escolar en el gran Córdoba (Argentina) 2001. Actas de las IX Jornadas Argentinas de Estudios de Población. Recuperado de http://www.aacademica.org/000-028/39.pdf

Instituto Nacional de Estadística (mayo, 2014). Encuesta de condiciones de vida (ECV2013). Recuperado de http://www.ine.es/prensa/np908.pdf

Izquierdo, T. (Coord.). (2010). Los nuevos retos del mercado laboral. Una perspectiva desde la orientación profesional. Úbeda: Amarantos.

Jiménez, T., Musitu, G. y Herrero, J. (2011). Familia y exclusión social en la adolescencia. En L. V. Amador y G. Musitu (Dirs.), Exclusión social y diversidad (pp. 143-168). México: Trillas.

Lépore, E. (2006). Exclusión social: en busca de su especificidad conceptual (versión preliminar). Recuperado de http://www.redadultosmayores.com.ar/buscador/files/ ARGEN015_Lepore.pdf

Morón, J.A. y Cobacho, M.L. (2009). Educación y promoción de la salud. Una mirada contextual. Murcia: Diego Marín.

Organización para la Cooperación y el Desarrollo Económico (2011). OECD Perspectives: Spain policies for a sustanaible recovery (OCDE Publicación No. 44686629). doi: 10.1787/9789264201736-en

Oxfam Intermón (2104). Gobernar para las élites. Secuestro democrático y desigualdad económica (Informe de investigación No. 178). Recuperado del sitio de Internet Oxfam: http://www.oxfam.org

Peña, D. (2010). Propuestas para la reforma de la universidad española. Madrid: Fundación Alternativas.

Pieck, E. (2001). Los jóvenes y el trabajo: La educación frente a la exclusión social. México: Universidad Iberoamericana.

Quintini, G. y Manfredi, T. (2009). Going separate ways? School to work transitions in the United States and Europe (OCDE Publicación No.90). doi: 10.1787/221717700447

Rodríguez, E. y Ballesteros, J. (2013). Crisis y contrato social: Los jóvenes en la sociedad del futuro. Recuperado del sitio de Internet Centro Reina Sofía sobre Adolescencia y Juventud de la Fundación de Ayuda contra la Drogadicción, Madrid: http://www. fad.es/publicaciones /resumen ejecutivo_crisis.pdf

Sánchez, J. (2013). De la pobreza a la exclusión: La lógica perversa del sistema imperante. Murcia: Foro Ignacio Ellacuría.

Stiglitz, J. E. (2003). El malestar en la globalización (Trad. C. Rodríguez; 2a ed.). Madrid: Santillana. (Reimpreso de Globalization and its discontent, por J. Stiglitz, Ed., 2002, Reino Unido: Penguin Books).

Stiglitz, J. E. (2012). El precio de la desigualdad: El 1\% de la población tiene lo que el 99\% necesita (Trad. A Pradera). Madrid: Taurus. (Reimpreso de The price of inequality, por J. Stiglitz, Ed., 2012, Reino Unido: Penguin Books). 
Tezanos, J. F. (2005). Exclusión social, democracia y ciudadanía económica. La libertad de los iguales. En J. García Molina (Coord.), Exclusión social, exclusión educativa. Lógicas contemporáneas (Vol. 8, pp. 47-59). Barcelona: Diálogos.

Wacquant, L.J.D. (2010). Castigar a los pobres: El gobierno neoliberal de la inseguridad social (Trad. M. Polo). Madrid: Gedisa. (Reimpreso de Punir les pauvres: Le nuveau goubernement do l'insécurité sociale, por L.J.D. Wacquant, Ed., 2004, Marsella, Francia: Agone).

Fecha de recepción: 30 de septiembre de 2015.

Fecha de revisión: 30 de septiembre de 2015.

Fecha de aceptación: 29 de marzo de 2016. 
